\title{
Multiple low-turbulence starless cores associated with intermediate- to high-mass star formation ${ }^{\star}$
}

\author{
H. Beuther and Th. Henning
}

\author{
Max-Planck-Institute for Astronomy, Königstuhl 17, 69117 Heidelberg, Germany \\ e-mail: [beuther; henning] @mpia.de
}

Received 10 March 2009 / Accepted 06 July 2009

\begin{abstract}
Aims. Characterizing the gas and dust properties prior to and in the neighborhood of active intermediate- to high-mass star formation. Methods. Two Infrared Dark Clouds (IRDCs) - IRDC 19175-4 and IRDC 19175-5 - that are located in the vicinity of the luminous massive star-forming region IRAS $19175+1357$, but that remain absorption features up to $70 \mu \mathrm{m}$ wavelength, were observed with the Plateau de Bure Interferometer in the $3.23 \mathrm{~mm}$ dust continuum as well as the $\mathrm{N}_{2} \mathrm{H}^{+}(1-0)$ and ${ }^{13} \mathrm{CS}(2-1)$ line emission.

Results. While IRDC $19175-4$ is clearly detected in the $3.23 \mathrm{~mm}$ continuum, the second source in the field, IRDC $19175-5$, is only barely observable above the $3 \sigma$ continuum detection threshold. However, the $\mathrm{N}_{2} \mathrm{H}^{+}(1-0)$ observations reveal 17 separate sub-sources in the vicinity of the two IRDCs. Most of them exhibit low levels of turbulence $\left(\Delta v \leq 1 \mathrm{~km} \mathrm{~s}^{-1}\right)$, indicating that the fragmentation process in these cores may be dominated by the interplay of thermal pressure and gravity, but not so much by turbulence. Combining the small line widths with the non-detection up to $70 \mu \mathrm{m}$ and the absence of other signs of star formation activity, most of these 17 cores with masses between sub-solar to $\sim 10 M_{\odot}$ are likely still in a starless phase. The $\mathrm{N}_{2} \mathrm{H}^{+}$column density analysis indicates significant abundance variations between the cores. Furthermore, we find a large CS depletion factor of the order 100. Although the strongest line and continuum peak is close to virial equilibrium, its slightly broader line width compared to the other cores is consistent with it being in a contraction phase potentially at the verge of star formation. Based on the $3.23 \mathrm{~mm}$ upper limits, the other cores may be gravitationally stable or even transient structures. The relative peak velocities between neighboring cores are usually below $1 \mathrm{~km} \mathrm{~s}^{-1}$, and we do not identify streaming motions along the filamentary structures. Average densities are between $10^{5}$ and $10^{6} \mathrm{~cm}^{-3}$ (one to two orders of magnitude larger than for example in the Pipe nebula) implying relatively small Jeans-lengths that are consistent with the observed core separations of the order $5000 \mathrm{AU}$. Environmental reasons potentially determining these values are discussed.

Conclusions. These observations show that multiple low- to intermediate-mass low-turbulence starless cores can exist in the proximity of more turbulent active intermediate- to high-mass star-forming regions. While masses and levels of turbulence are consistent with low-mass starless core regions, other parameters like the densities or Jeans-lengths differ considerably. This may be due to environmental effects. The quest for high-mass starless cores prior to any star formation activity remains open.
\end{abstract}

Key words. stars: formation - stars: individual: IRDC 19175-4, IRDC 19175-5 - techniques: interferometric - line: formation - line: profiles - turbulence

\section{Introduction}

Although our understanding of massive star formation has improved tremendously over the last decade, most progress was made toward relatively evolved regions like high-mass protostellar objects (HMPOs), hot molecular cores (HMCs) or ultracompact HII regions (UCHIIs), see for example several recent reviews like Beuther et al. (2007); Bonnell et al. (2007); Cesaroni et al. (2007); Hoare et al. (2007); Zinnecker \& Yorke (2007). Toward even earlier evolutionary stages prior or at the onset of massive star formation, in recent years significant progresses have been made in identifying large samples of infrared dark clouds (IRDCs) via absorption studies in the mid-infrared with the MSX, ISO and Spitzer satellites (e.g., Egan et al. 1998; Bacmann et al. 2000; Simon et al. 2006; Jackson et al. 2008). Such IRDCs are expected to harbor genuine high-mass starless cores (HMSCs) as well as high-mass cores with embedded lowto intermediate-mass protostars in the early accretion phase (e.g., Forbrich et al. 2004; Klein et al. 2005; Sridharan et al. 2005;

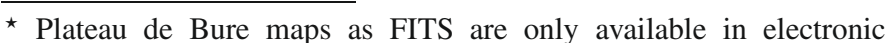
form at the CDS via anonymous ftp to cdsarc.u-strasbg.fr (130.79.128.5) or via

http://cdsweb.u-strasbg.fr/cgi-bin/qcat?J/A+A/503/859
Rathborne et al. 2005, 2006; Beuther \& Steinacker 2007; Beuther \& Sridharan 2007). While the samples contain more than 10000 IRDCs, it is interesting to note that from the published higher spatial resolution case studies (of the order 20) none is a real HMSC but all have identified embedded accreting protostars (e.g., Beuther et al. 2005; Beuther \& Steinacker 2007; Birkmann et al. 2006; Ormel et al. 2005; Pillai et al. 2006; Rathborne et al. 2005, 2008a; Forbrich et al. 2009). Hence, a genuine HMSC has not yet been unambiguously identified in the literature.

To overcome this obvious missing link, here we report highspatial-resolution observations and analysis of two excellent HMSC candidates in the dust continuum and the $\mathrm{N}_{2} \mathrm{H}^{+}$line emission to investigate their physical properties prior to any star formation activity. $\mathrm{N}_{2} \mathrm{H}^{+}$is known to be strong in low-mass starless cores (e.g., Bergin et al. 2002; Tafalla et al. 2004; Chen et al. 2007) as well as massive IRDCs with already embedded, very young protostars (e.g., Beuther et al. 2005). Furthermore, it is a good chemical tracer of the cold early evolutionary phase because it does not deplete much onto grains at low temperatures but it starts getting destroyed when the core heats up releasing the $\mathrm{CO}$ from the dust grains (which was frozen onto them during earlier cold phases) that subsequently reacts with $\mathrm{N}_{2} \mathrm{H}^{+}$in the 


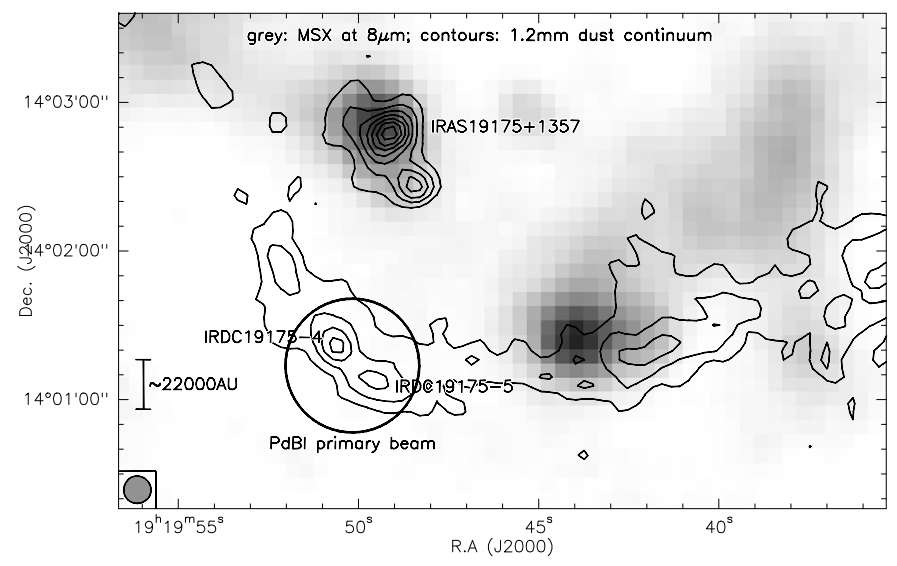

Fig. 1. Large-scale emission in the environment of the sources IRDC 19175-4, IRDC 19175-5 and IRAS 19175+1357. The grey-scale shows the MSX $8 \mu \mathrm{m}$ mid-infrared emission, and the contours outline the $1.2 \mathrm{~mm}$ continuum emission from Beuther et al. (2002) in steps of $20 \mathrm{mJybeam}^{-1}\left(3 \sigma \mathrm{rms} \sim 15 \mathrm{mJy}\right.$ beam $\left.^{-1}\right)$. The circle presents the PdBI primary beam size of $\sim 54^{\prime \prime}$ encompassing the two target regions. A scale-bar and the IRAM $30 \mathrm{~m}$ beam size of the $1.2 \mathrm{~mm}$ continuum observations are shown at the bottom left.

gas-phase chemistry (Bergin \& Langer 1997). While the dust continuum emission pinpoints the locations of the densest gas and dust components, the line emission will be used to determine the gas kinematics of the region.

The two HMSC candidate sources are located in the vicinity of the High-Mass Protostellar Object (HMPO) IRAS 19175+1357. During our initial study of the dust continuum emission of a sample of HMPOs (Sridharan et al. 2002; Beuther et al. 2002), we serendipitously detected mm continuum peaks offset from the IRAS sources that turned out by comparison with the MSX database to be associated with IRDCs (Sridharan et al. 2005). The average $\mathrm{NH}_{3}$ temperatures and line widths of these objects are significantly smaller than those from typical HMPOs and UCHIIs, hence putting them at an earlier evolutionary stage. However, follow-up $\mathrm{SiO}(2-1)$ observations revealed $\mathrm{SiO}$ line wing emission toward $\sim 40 \%$ of the sample, indicating that star formation has started in at least that fraction of sources (Beuther \& Sridharan 2007).

To identify a candidate HMSC for this sample with the given datasets, we require the source to have (a) mm continuum emission; (b) no detectable $\mathrm{SiO}$ emission; (c) narrow $\mathrm{NH}_{3}$ and $\mathrm{H}^{13} \mathrm{CO}^{+}$line width; (d) low $\mathrm{NH}_{3}$ temperatures and (e) no detections in the Spitzer IRAC and MIPS 24 and $70 \mu \mathrm{m}$ bands. Here, we select from the original sample two particularly promising HMSC candidates that fulfill these criteria and even fit within one PdBI $3 \mathrm{~mm}$ primary beam (Fig. 1).

The two sources IRDC 19175-4 and IRDC 19175-5 with a projected separation of $\sim 22000 \mathrm{AU}$ are part of the larger-scale filamentary star-forming region associated with IRAS $19175+1357$ (Fig. 1) at an estimated distance of $\sim 1.1 \mathrm{kpc}$ (Sridharan et al. 2005). The luminosity of the main IRAS source in Fig. 1 is approximately $10^{3} L_{\odot}$, lower than originally estimated based on a wrong distance in Sridharan et al. (2002). Sridharan et al. (2002) found weak cm continuum emission indicative of ionized gas in the vicinity of the IRAS 19175+1357 source. However, no maser emission or strong CO line wings were detected. The latter indicates either only weak or no outflow activity anymore toward this main source, or the outflow could be oriented close to the plane of the sky. Since the $v_{\mathrm{lsr}}$ of the main IRAS source and the two IRDCs are approximately the same (6.9 versus $7.7 \mathrm{~km} \mathrm{~s}^{-1}$ ), we can safely assume that both regions are at about the same distance. Hence their projected separation of $\sim 0.45 \mathrm{pc}$ is a good approximation of their real separation. Although we cannot proof that the IRAS source directly influences the two IRDCs, both regions should nevertheless stem from the same larger gas clump that originally fragmented into the already more evolved IRAS source and the younger IRDCs.

Figure 2 presents an overlay of all IRAC and the MIPS 24 and $70 \mu \mathrm{m}$ bands (Churchwell et al. 2009; Carey et al. 2009) with the dust continuum emission towards IRDC 19175-4 and IRDC 19175-5, and both regions remain dark even up to $70 \mu \mathrm{m}$. The $24 \mu \mathrm{m}$ absorption feature toward IRDC $19175-5$ is a bit more pronounced than toward IRDC 19175-4. We detected no $\mathrm{SiO}$ emission. Their $\mathrm{NH}_{3}$ single-dish line widths are $\sim 1.5 \mathrm{~km} \mathrm{~s}^{-1}$, and their derived $\mathrm{NH}_{3}$ rotation temperatures are of the order $15 \mathrm{~K}$ (Sridharan et al. 2002; Beuther \& Sridharan 2007). All these characteristics make the two sources ideal HMSC candidates and hence well-suited targets to investigate the physical properties prior to the onset of star formation.

Assuming optically thin emission from cold dust at $15 \mathrm{~K}$ and a distance of $1.1 \mathrm{kpc}$ with a gas-to-dust mass ratio of 186 (following Draine et al. 2007; Jenkins 2004), the integrated $1.2 \mathrm{~mm}$ emission from the two region IRDC 19175-4 and IRDC 19175-5 of $620 \mathrm{mJy}$ (Beuther et al. 2002) corresponds to approximately $87 M_{\odot}$ of gas mass, fairly equally divided between both sources. While these gas masses are not of the order $1000 M_{\odot}$ like some better known massive star-forming regions, the gas reservoir is large enough to potentially form intermediate-mass B-type stars.

\section{Observations}

We observed IRDC 19175-4 and IRDC19175-5 with the Plateau de Bure Interferometer during two nights in August 2007 and April 2008 at $93 \mathrm{GHz}$ in the $\mathrm{C}$ and D configurations covering projected baselines between approximately 13 and $180 \mathrm{~m}$. The $3 \mathrm{~mm}$ receivers were tuned to $92.835 \mathrm{GHz}$ in the lower sideband covering the $\mathrm{N}_{2} \mathrm{H}^{+}(1-0)$ and the ${ }^{13} \mathrm{CS}(2-1)$ lines as well as the $3.23 \mathrm{~mm}$ continuum emission. The phase noise was always lower than $30^{\circ}$ and mostly lower than $20^{\circ}$. For continuum measurements we placed six $320 \mathrm{MHz}$ correlator units in the band, the spectral lines were excluded in averaging the units to produce the final continuum image. Temporal fluctuations of amplitude and phase were calibrated with frequent observations of the quasars 1923-201 and 1827+062. The amplitude scale was derived from measurements of MWC349. We estimate the final flux accuracy to be correct to within $\sim 15 \%$. The phase reference center is RA[J2000] $19^{\mathrm{h}} 19^{\mathrm{m}} 50.169^{\mathrm{s}}$ and Dec[J2000] $14^{\circ} 01^{\prime} 13.75^{\prime \prime}$, and the velocity of rest $v_{\mathrm{lsr}}$ is $7.7 \mathrm{~km} \mathrm{~s}^{-1}$. The synthesized beam of the continuum data with a robust weighting is $3.5^{\prime \prime} \times 2.8^{\prime \prime}\left(\mathrm{PA} 28^{\circ}\right.$ east of north) with a $3 \sigma$ continuum $\mathrm{rms}$ of $0.16 \mathrm{mJy}_{\text {beam }}^{-1}$. The $3 \sigma \mathrm{rms}$ of the $\mathrm{N}_{2} \mathrm{H}^{+}(1-0)$ data measured from an emission-free channel with a spectral resolution of $0.2 \mathrm{~km} \mathrm{~s}^{-1}$ is $15 \mathrm{mJy}$ beam ${ }^{-1}$. The $3 \sigma \mathrm{rms}$ of the ${ }^{13} \mathrm{CS}(2-1)$ data measured from an emission-free channel with a spectral resolution of $0.5 \mathrm{~km} \mathrm{~s}^{-1}$ is $10 \mathrm{mJy}$ beam $^{-1}$.

These mm observations are complemented with the midinfrared SPITZER data from the GLIMPSE and MIPSGAL surveys of the Galactic plane using the IRAC camera centered at 3.6, 4.5, 5.8 and $8.0 \mu \mathrm{m}$ and the MIPS camera centered at 24 and $70 \mu \mathrm{m}$ (Werner et al. 2004; Fazio et al. 2004; Benjamin et al. 2003; Rieke et al. 2004; Carey et al. 2005). 


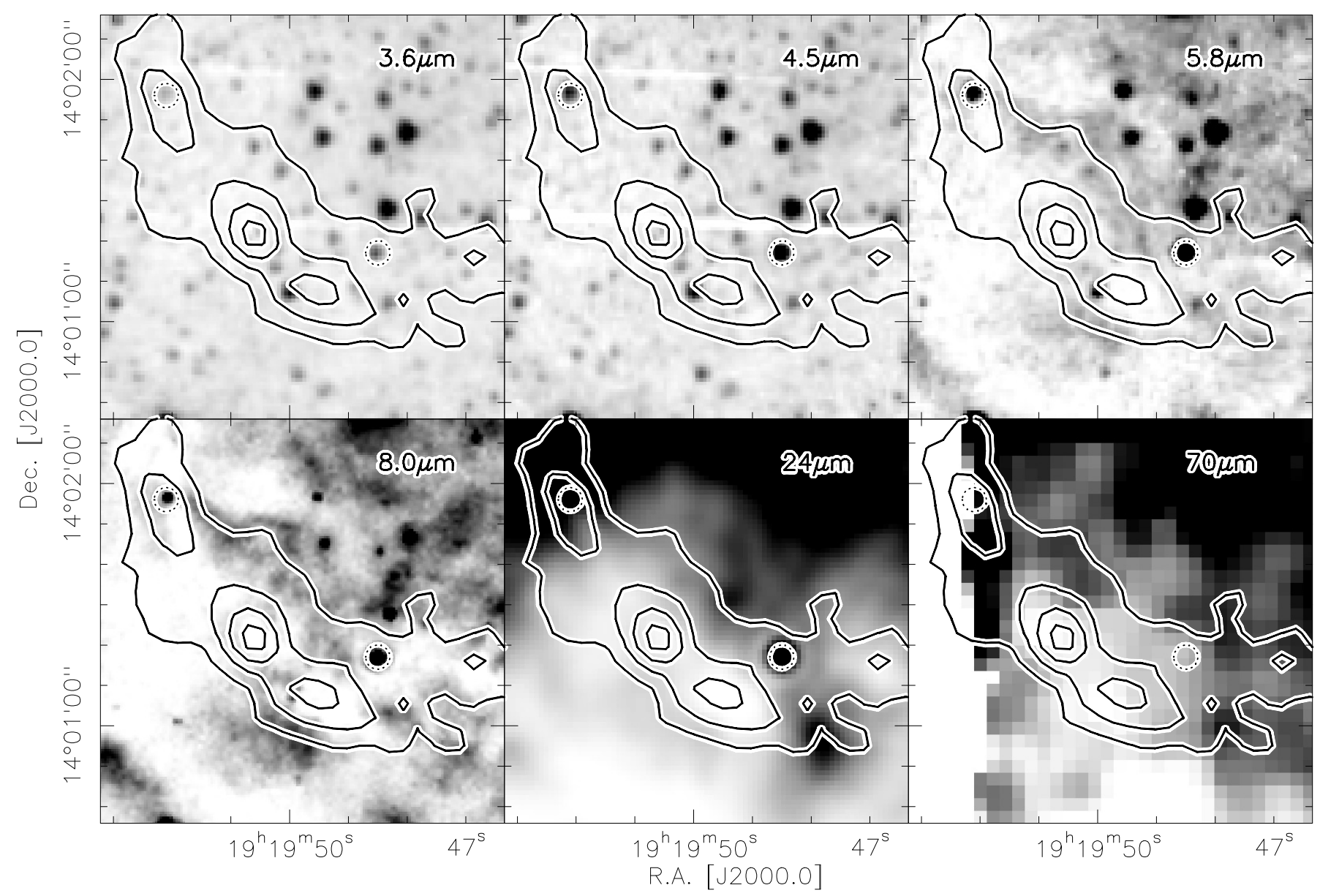

Fig. 2. Zoom into the central region of the two target sources. Like Fig. 1, the contours show the $1.2 \mathrm{~mm}$ dust continuum emission (Beuther et al. 2002), and the grey-scale presents the mid- to far-infrared emission as observed with the Spitzer Space Telescope. From top-left to bottom-right, the 6 panels show the Spitzer bands centered at 3.6, 4.5, 5.8, 8.0, 24 and $70 \mu \mathrm{m}$, as marked in each panel. The dotted circles in each panel mark the only two Spitzer sources that were identified as class0/I candidates (see main text).

\section{Results}

\subsection{Millimeter continuum emission and class $0 / 1$ sources identified via the Spitzer data}

Figure 3 presents an overlay of the $1.2 \mathrm{~mm}$ dust continuum data from the IRAM $30 \mathrm{~m}$ telescope (Beuther et al. 2002) with the new high-spatial-resolution $3.23 \mathrm{~mm}$ data obtained now with the PdBI. While the north-eastern peak (IRDC 19175-4) is clearly detected also in the new $3.23 \mathrm{~mm}$ data, the south-western peak (IRDC 19175-5) is a marginal detection at less than $5 \sigma$ with $0.26 \mathrm{mJy} \mathrm{beam}^{-1}$. The peak and integrated $3.23 \mathrm{~mm}$ fluxes of IRDC $19175-4$ are $1.09 \mathrm{mJy}^{-1} \mathrm{beam}^{-1}$ and $1.8 \mathrm{mJy}$, respectively. Following Hildebrand (1983) and Beuther et al. (2002), assuming optically thin dust continuum emission with a gas-to-dust mass ratio of 186 (Draine et al. 2007; Jenkins 2004), a dust opacity index $\beta=2$ (corresponding to a typical dust distribution as outlined in Mathis et al. 1977; Ossenkopf \& Henning 1994) and a temperature of $15 \mathrm{~K}$ (Sridharan et al. 2005), we derive a gas mass and column density of $\sim 10 M_{\odot}$ and $\sim 1.8 \times 10^{24} \mathrm{~cm}^{-2}$, respectively. The latter corresponds to a visual extinction $A_{v}$ of $\sim 1900 \mathrm{mag}$ (Frerking et al. 1982). Our $3 \sigma$ continuum sensitivity corresponds with the given parameters to $3 \sigma$ mass and column density sensitivities of $0.9 M_{\odot}$ and $1.7 \times 10^{23} \mathrm{~cm}^{-2}$. Comparing the mass derived from the PdBI data of IRDC $19175-$ 4 of $\sim 10 M_{\odot}$ with the single-dish estimated mass of $87 M_{\odot}$, approximately $90 \%$ of the flux has been filtered out by the interferometer observations.

Although the mm continuum peaks are infrared dark up to $70 \mu \mathrm{m}$ emission, Fig. 2 shows several Spitzer sources within the field of view. Employing the available IRAC GLIMPSE point source catalog (Churchwell et al. 2009 and http://irsa.ipac.caltech.edu/) and adopting the Spitzer color criteria by Allen et al. (2004), we tried to identify possible class $0 / \mathrm{I}$ and class II protostellar candidates around the discussed IRDCs (see also Fang et al. 2009). Interestingly, we do not find any class II source and only 2 class 0/I candidates close to IRDC 19175-4 and IRDC 19175-5 (see dotted circles in Fig. 2). While these two class 0/I candidates are clearly within the vicinity of the IRDCs, almost all of the remaining sources in the field of view are either more evolved and hence do not fit the Spitzer color criteria, or they are entirely unrelated foreand/or background sources. Identifying only two young protostellar candidates with locations not toward the mm continuum peaks further stresses the youth of the target regions.

\section{2. $\mathrm{N}_{2} \mathrm{H}^{+}(1-0)$ emission}

Figures 4-6 present the $\mathrm{N}_{2} \mathrm{H}^{+}(1-0)$ data as integrated intensity maps, a channel map and the deduced 1st and 2nd moment maps (intensity-weighted peak velocities and line widths). For the channel and moment maps, we use the isolated 


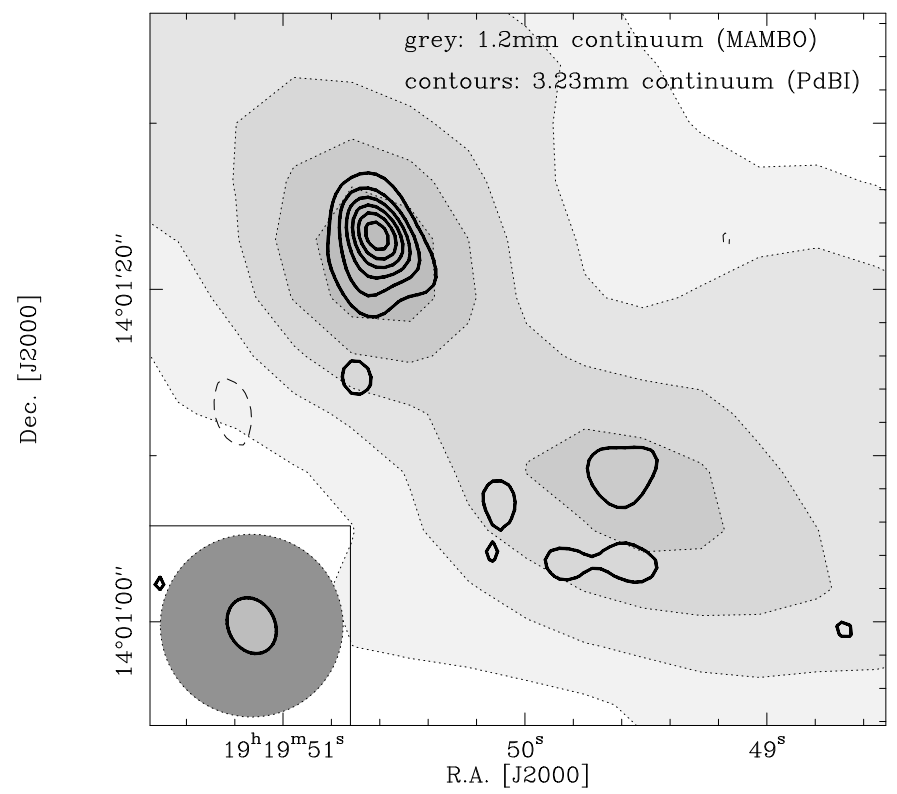

Fig. 3. Millimeter continuum emission toward IRDC 19175-4 and IRDC19175-5. The grey-scale with dotted contours presents the single-dish $1.2 \mathrm{~mm}$ continuum observations with the MAMBO array mounted on the IRAM $30 \mathrm{~m}$ telescope contoured at the $3 \sigma$ levels of $15 \mathrm{mJy}$ beam $^{-1}$ (Fig. 1 and Beuther et al. 2002), and the thick contours show the new $3.23 \mathrm{~mm}$ data from the PdBI observed for this project with $3 \sigma$ contours of $0.16 \mathrm{mJy}$ beam $^{-1}$. Full and dashed contours present emission and negative features due to the insufficient uv-coverage, respectively. In the bottom-left corner, the beam of the $1.2 \mathrm{~mm}$ singledish observations is shown as the large circle, and the synthesized PdBI beam as smaller ellipse.

$\mathrm{N}_{2} \mathrm{H}^{+}(1-0)(\mathrm{F} 1, F=0,1 \rightarrow 1,2)$ hyperfine emission component at $93176.2650 \mathrm{MHz}$ (Caselli et al. 1995) which is blue-shifted by $-8.0 \mathrm{~km} \mathrm{~s}^{-1}$ with respect to the central component because it is a spectrally isolated line without further line-blending and mostly optically thin. In strong contrast to the relatively simple dust continuum structure, the $\mathrm{N}_{2} \mathrm{H}^{+}$data now reveal a large amount of separate emission peaks, 17 in number. Although the general structure of the two integrated maps of the main and satellite hyperfine structure lines in Fig. 4 is very similar, small discrepancies arise because of different sensitivities and opacities. While $\mathrm{N}_{2} \mathrm{H}^{+}$peaks $10,11,14,15$ and 17 are mainly detected in the main hyperfine structure line most likely because it can trace lower column density structures, other peaks are offset between the two integrated images. For example the $\mathrm{N}_{2} \mathrm{H}^{+}$peaks 7 and 8 are clearly separated in the satellite hyperfine structure line map, whereas it appears like a single peak in the main hyperfine line. This difference likely arises from the higher optical depth of the main line. For our source-labeling we refer to the positions from the isolated satellite hyperfine structure line where they were clearly detected. For the remaining peaks, the positions are extracted from the main hyperfine line. While the strongest $\mathrm{N}_{2} \mathrm{H}^{+}$peak number 1 is associated with the strongest mm continuum peak, the second $\mathrm{mm}$ peak has no directly associated $\mathrm{N}_{2} \mathrm{H}^{+}$feature, rather the $\mathrm{N}_{2} \mathrm{H}^{+}$peaks 4 and 5 are lying at the edge of the mm continuum emission. Figure 7 presents a few example $\mathrm{N}_{2} \mathrm{H}^{+}$spectra, and Table 1 shows the corresponding spectral parameters (peak line temperature $T_{\text {peak }}$, peak velocity $v$, line width $\Delta v$ and total optical depth $\tau$ of all hyperfine components) we derived from fitting the complete $\mathrm{N}_{2} \mathrm{H}^{+}(1-0)$ hyperfine structure within CLASS (part of the GILDAS software

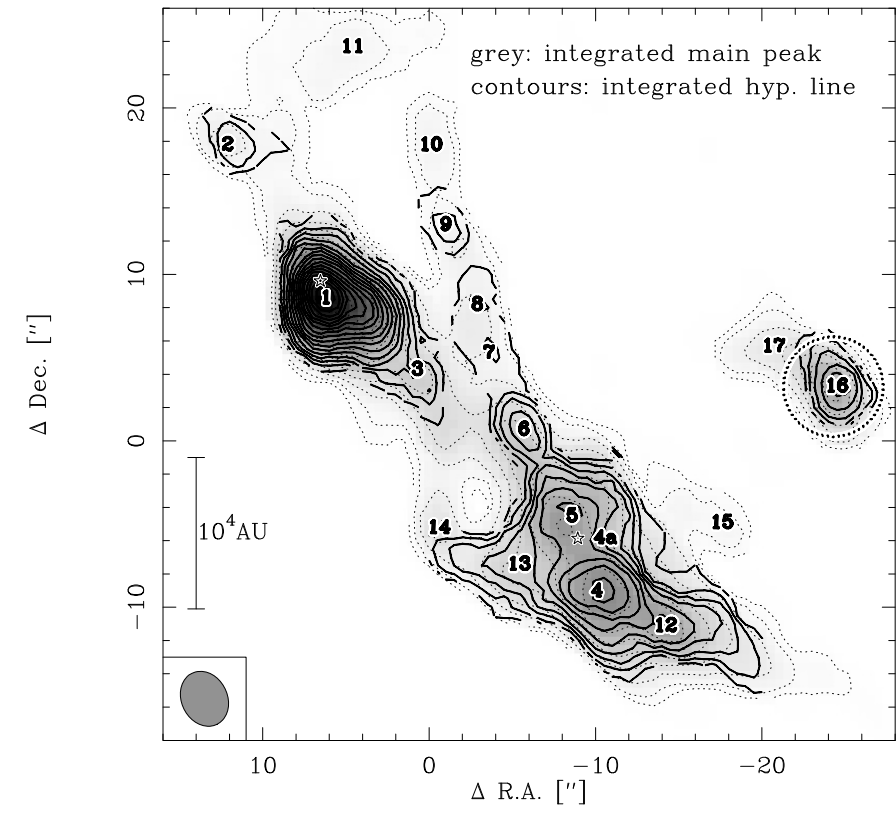

Fig. 4. The thick contours present the integrated emission of the isolated $\mathrm{N}_{2} \mathrm{H}^{+}(1-0)(\mathrm{F} 1, F=0,1 \rightarrow 1,2)$ hyperfine emission component at $93176.2650 \mathrm{MHz}$ (Caselli et al. 1995) which is blue-shifted by $-8.0 \mathrm{~km} \mathrm{~s}^{-1}$ with respect to the central component (see Fig. 7). The contour levels are in steps of $10 \mathrm{mJy}_{\text {beam }} \mathrm{km} \mathrm{s}^{-1}$. The grey-scale with dotted contours shows the integrated emission of the central few hyperfine components around the $v_{\mathrm{lsr}}$ with contour levels of 20,40 and then continuing in $40 \mathrm{mJy}^{-1} \mathrm{bm}^{-1} \mathrm{~km} \mathrm{~s}^{-1}$ steps. To produce the integrated intensity (or 0th moment) maps, data were clipped below $5 \sigma$ corresponding to $25 \mathrm{mJy}_{\text {beam }}^{-1}$ for a channel width of $0.2 \mathrm{~km} \mathrm{~s}^{-1}$. The corresponding integration regimes are 6.3 to $8.9 \mathrm{~km} \mathrm{~s}^{-1}$ for the isolated hyperfine structure line and 5.5 to $10.7 \mathrm{~km} \mathrm{~s}^{-1}$ for the blended central line, respectively. The stars mark the positions of the main mm continuum peaks (Figs. 1 and 3), and the synthesized beam and scale-bar are shown at the bottom-left. The numbers mark the $17 \mathrm{~N}_{2} \mathrm{H}^{+}(1-0)$ emission peaks discussed in the main text and Table 1 . The dotted circle associated with the $\mathrm{N}_{2} \mathrm{H}^{+}$peak 16 marks the position of the western Spitzer class 0/I source. The offsets are given with respect to the phase center.

package ${ }^{1}$ ). While the $\mathrm{N}_{2} \mathrm{H}^{+}$peak 16 is spatially associated with the western Spitzer class 0/I source (Figs. 2 and 6), no other $\mathrm{N}_{2} \mathrm{H}^{+}$peak has a Spitzer-identifiable young protostellar counterpart. Except of peak 16, all $\mathrm{N}_{2} \mathrm{H}^{+}$peaks are good candidates for being genuine starless cores.

The sub-structure of the cores exhibits an elongated and filamentary structure. The filament associated with the $\mathrm{N}_{2} \mathrm{H}^{+}$peaks 6 to 10 has a linear extend of $\sim 25000 \mathrm{AU}$ with a length-to-width ratio exceeding 5 . The average separation between the five cores is $\sim 5000$ AU. Following Stahler \& Palla (2005) we can calculate the Jeans-length

$\lambda_{J}=\left(\frac{\pi a^{2}}{G \rho}\right)^{1 / 2}=0.19 \mathrm{pc}\left(\frac{T}{10 \mathrm{~K}}\right)^{1 / 2}\left(\frac{n_{\mathrm{H}_{2}}}{10^{4} \mathrm{~cm}^{-3}}\right)^{-1 / 2}$

with the sound speed $a$, the gravitational constant $G$ and the density $\rho$. What density do we expect for the cores? The critical density of the $\mathrm{N}_{2} \mathrm{H}^{+}(1-0)$ transition at the given low temperature is $\sim 1.5 \times 10^{5} \mathrm{~cm}^{-3}$. In addition to this, we can calculate average densities for each core converting the $\mathrm{N}_{2} \mathrm{H}^{+}$column densities (see Sect. 3.3) to $\mathrm{H}_{2}$ column densities assuming a typical $\mathrm{N}_{2} \mathrm{H}^{+}$ to $\mathrm{H}_{2}$ abundance ratio of $3 \times 10^{-10}$ (Caselli et al. 2002a), and furthermore assuming in spherical geometry that the 3rd dimension

${ }^{1}$ http://www.iram.fr/IRAMFR/GILDAS 


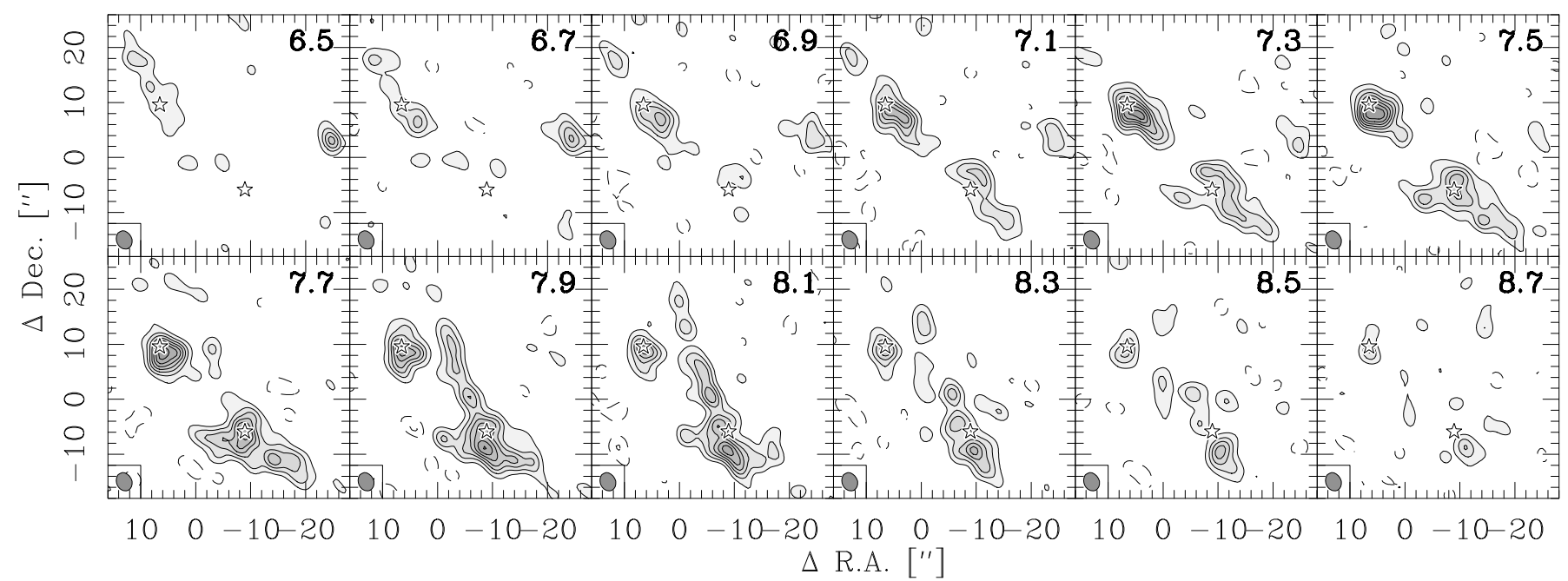

Fig. 5. Channel map of the isolated $\mathrm{N}_{2} \mathrm{H}^{+}(1-0)(\mathrm{F} 1, F=0,1 \rightarrow 1,2)$ hyperfine emission component at $93176.2650 \mathrm{MHz}$ (Caselli et al. 1995) which is blue-shifted by $-8.0 \mathrm{~km} \mathrm{~s}^{-1}$ with respect to the central component (see Fig. 7). The velocities as marked in the top-right corners of each panel are shifted to the velocity of rest. The grey-scale and contours are done in $3 \sigma$ steps of $15 \mathrm{mJy}_{\text {beam }}^{-1}$ (full and dashed contours positive and negative, respectively). The two stars mark the main two mm continuum sources (Figs. 1 and 3), the numbers in the top-right corners show the central velocities of each channel, and the synthesized beam is plotted at the bottom-left of each panel. The offsets are given with respect to the phase center.

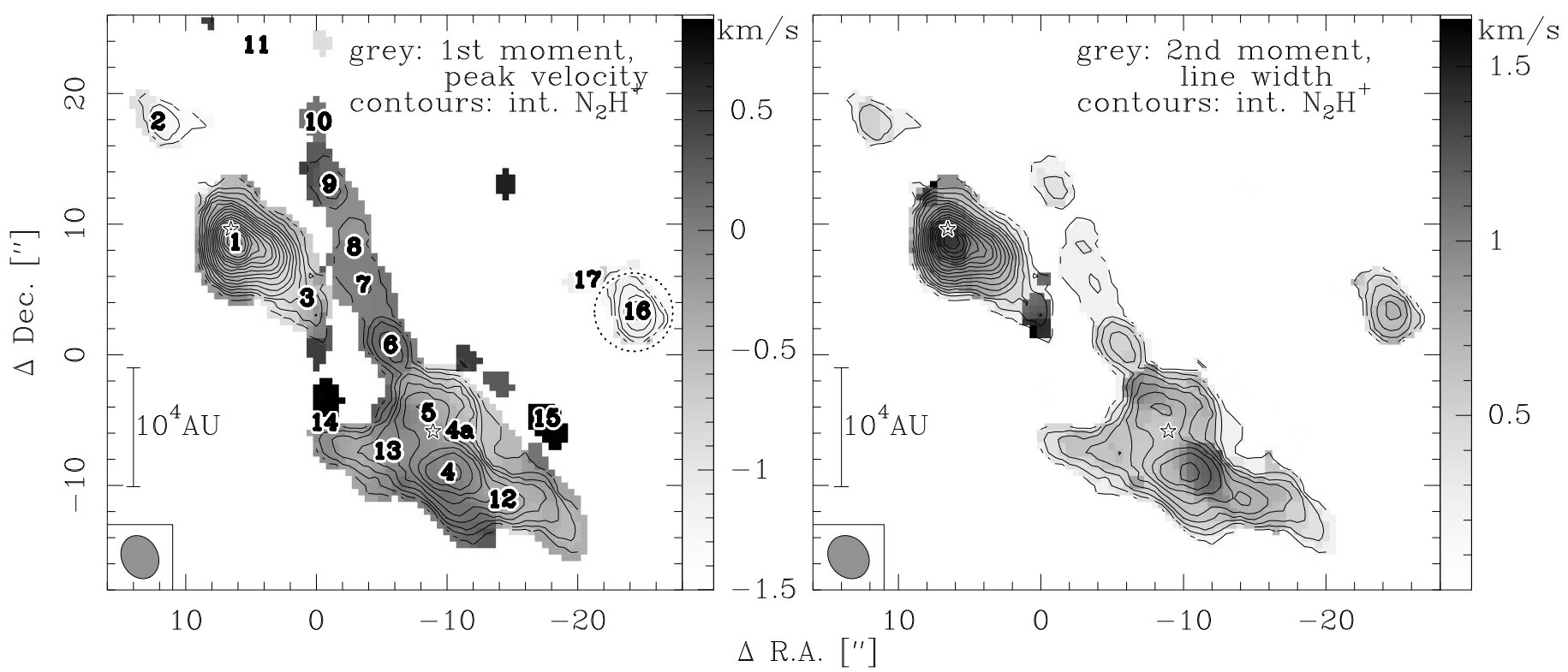

Fig. 6. Moment maps of the $\mathrm{N}_{2} \mathrm{H}^{+}(1-0)$ emission. The grey-scale in the left and right panels present the 1st and 2nd moments, respectively (intensity-weighted peak velocities and line widths). The contours in both panels show the 0th moment or integrated intensity maps which were produced from the channel map in Fig. 5 via clipping all data below $5 \sigma$ corresponding to $25 \mathrm{mJy} \mathrm{beam}^{-1}$ for a channel width of $0.2 \mathrm{~km} \mathrm{~s}^{-1}$. The contour levels are done in steps of $10 \mathrm{mJy}$ beam ${ }^{-1} \mathrm{~km} \mathrm{~s}^{-1}$. The maps were produced from the isolated $\mathrm{N}_{2} \mathrm{H}^{+}(1-0)(\mathrm{F} 1, F=0,1 \rightarrow 1,2)$ hyperfine emission component $\left(-8.0 \mathrm{~km} \mathrm{~s}^{-1}\right.$ offset from the central component) with an integration regime corresponding to 6.3 to $8.9 \mathrm{~km} \mathrm{~s}^{-1}\left(v_{\mathrm{lsr}}=7.7 \mathrm{~km} \mathrm{~s}^{-1}\right)$. The stars mark the positions of the main mm continuum peaks (Figs. 1 and 3), and the synthesized beam and scale-bar are shown at the bottom-left of each panel. The numbers in the left panel mark the $17 \mathrm{~N}_{2} \mathrm{H}^{+}(1-0)$ emission peaks discussed in the main text and Table 1 . The dotted circle associated with the $\mathrm{N}_{2} \mathrm{H}^{+}$peak 16 marks the position of the western Spitzer class 0/I source. The offsets are given with respect to the phase center.

along the line of sight corresponds to the synthesized beam for the compact cores. Table 1 lists the derived densities for all cores estimated this way. The range of average densities varies by about one order of magnitude between $2 \times 10^{5}$ and $1.9 \times 10^{6} \mathrm{~cm}^{-3}$. For the cores 6 to 10 along the filament, the average density is about $\sim 6 \times 10^{5} \mathrm{~cm}^{-3}$, implying that the peak densities should be even higher. Hence, we can estimate the Jeans-length at densities of $5 \times 10^{5}$ and $10^{6} \mathrm{~cm}^{-3}$ with a typical temperature of $15 \mathrm{~K}$. This brackets the corresponding Jeans-lengths approximately between $\sim 4800$ and $\sim 6800$ AU above which the filament should become unstable. Interestingly, the measured core separation corresponds well to this Jeans-length indicating that the cores could be stable against further fragmentation.

While the broadest line (still only $1.8 \mathrm{~km} \mathrm{~s}^{-1}$ full width half maximum) is associated with the main $\mathrm{mm}$ continuum peak, all other peak positions exhibit line widths close to or below $1 \mathrm{~km} \mathrm{~s}^{-1}$. The $\mathrm{N}_{2} \mathrm{H}^{+}(1-0)$ thermal line width at $15 \mathrm{~K}$ is $\sim 0.15 \mathrm{~km} \mathrm{~s}^{-1}$. Since the spectral resolution of our final $\mathrm{N}_{2} \mathrm{H}^{+}(1-0)$ data cube $\left(0.2 \mathrm{~km} \mathrm{~s}^{-1}\right)$ is insufficient to actually resolve the thermal line width, it is difficult to accurately constrain 

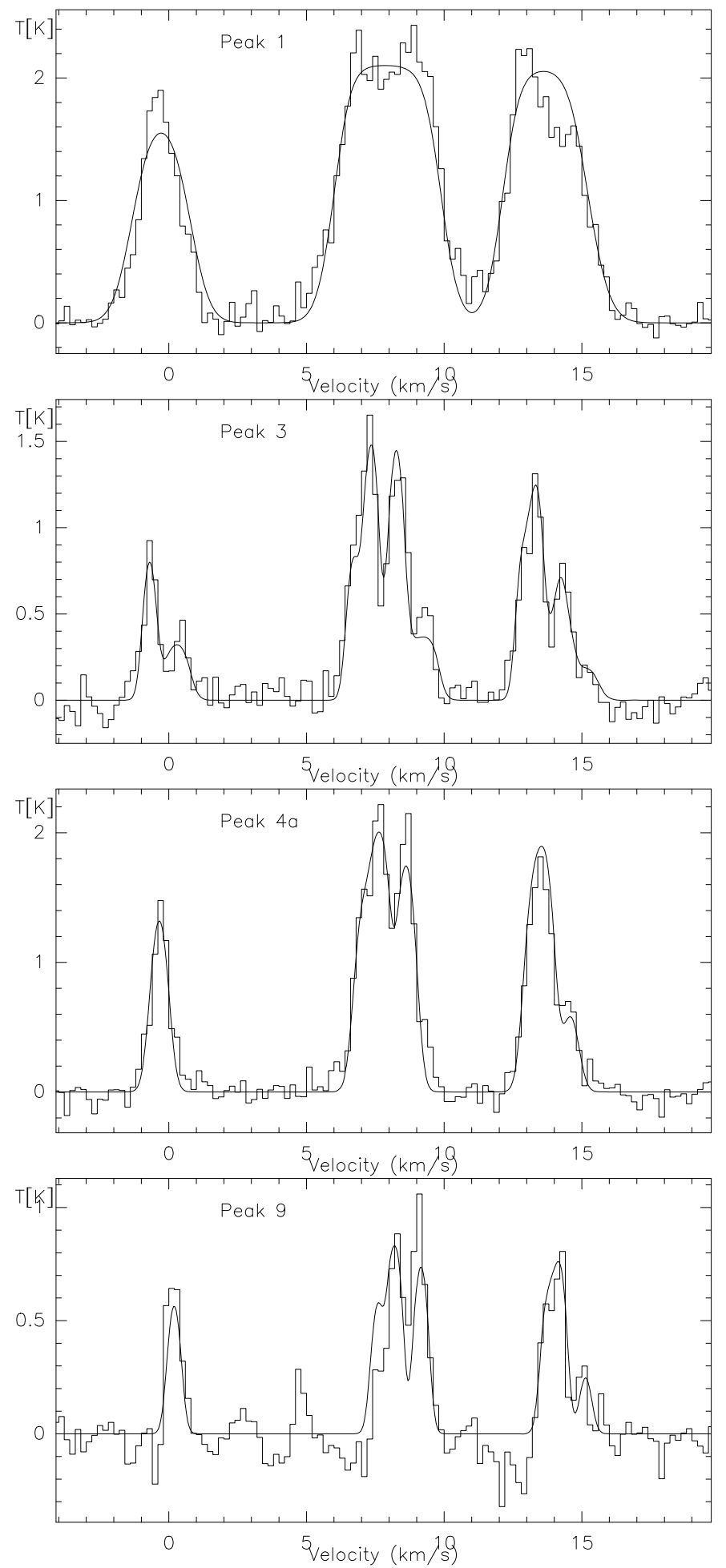

Fig. 7. Example $\mathrm{N}_{2} \mathrm{H}^{+}(1-0)$ spectra extracted toward the positions labeled in each panel (see Fig. 6 and Table 1). The full lines show the fits with the parameters listed in Table 1 . The $y$-axis shows the brightness temperature.

whether the narrowest measured line widths (Table 1) close to our spectral resolution (less than 3 spectral resolution elements across the line width $\Delta v$ ) are not rather upper limits to real line widths closer to thermal.

The relative peak velocities between close-by neighboring sub-cores is usually below $1 \mathrm{~km} \mathrm{~s}^{-1}$, and we do not identify any clear velocity gradient or streaming motions along the filamentary structures associated with the $\mathrm{N}_{2} \mathrm{H}^{+}$peak positions (see Fig. 6 and Table 1).

\section{3. $\mathrm{N}_{2} \mathrm{H}^{+}$column densities and abundances}

Using the line intensities and optical depths from our $\mathrm{N}_{2} \mathrm{H}^{+}(1-0)$ fitting, we can estimate the $\mathrm{N}_{2} \mathrm{H}^{+}$column densities for all cores (e.g, Caselli et al. 2002a). The resulting values between $0.3 \times 10^{13}$ and $6.6 \times 10^{13} \mathrm{~cm}^{-2}$ are also listed in Table 1 . With a typical $\mathrm{N}_{2} \mathrm{H}^{+} / \mathrm{H}_{2}$ abundance ratio of $3 \times 10^{-10}$ (Caselli et al. 2002a), this corresponds to an $\mathrm{H}_{2}$ column density regime between roughly $2 \times 10^{22}$ and $2 \times 10^{23} \mathrm{~cm}^{-1}$ (or visual extinctions $A_{v}$ between 20 and 200, Frerking et al. 1982), largely below our $\mathrm{H}_{2} 3 \sigma$ column density limit of $1.7 \times 10^{23} \mathrm{~cm}^{-2}$ derived from the $3.23 \mathrm{~mm}$ dust continuum emission (see Sect. 3.1). However, taking our derived $\mathrm{H}_{2}$ and $\mathrm{N}_{2} \mathrm{H}^{+}$column densities at face value, we derive an order of magnitude lower abundance of $\sim 3.7 \times 10^{-11}$ for the main $3.23 \mathrm{~mm}$ continuum and $\mathrm{N}_{2} \mathrm{H}^{+}$peak 1 associated with IRDC 19175-4. Assuming that this abundance could hold for all $\mathrm{N}_{2} \mathrm{H}^{+}(1-0)$ cores, the range of corresponding $\mathrm{H}_{2}$ column densities would be an order of magnitude higher, roughly between $2 \times 10^{23}$ and $2 \times 10^{24} \mathrm{~cm}^{-2}$. These values nearly all were above our $\mathrm{H}_{2} 3 \sigma$ column density limit of $1.7 \times 10^{23} \mathrm{~cm}^{-2}$ derived from the $3.23 \mathrm{~mm}$ continuum emission. Although all these estimates are prone to many uncertainties, they indicate that even during such early evolutionary stages the gas phase abundances are not uniform throughout the core. The source with the broadest line width, and hence likely the most evolved of the cores (peak 1 in IRDC 19175-4), shows the lowest abundance compared to the other less turbulent cores. While Tafalla \& Santiago (2004) argued in the case of the low-mass starless core L1571E that particular low $\mathrm{N}_{2} \mathrm{H}^{+}$abundances could also be due to very young evolutionary stages (see also Aikawa et al. 2003), the increased line width toward our main core makes this an unlikely scenario. Rather in contrast, increased densities in more evolved stages of core collapse (like those inferred here, see Table 1) increase the recombination rate and hence lower the $\mathrm{N}_{2} \mathrm{H}^{+}$abundance (e.g, Bergin \& Langer 1997). This appears the more likely scenario for the cores we observe here.

\section{4. ${ }^{13} \mathrm{CS}(2-1)$ emission}

In addition to the $\mathrm{N}_{2} \mathrm{H}^{+}(1-0)$ emission, our spectral setup also covered the ${ }^{13} \mathrm{CS}(2-1)$ line. However, although detected, the ${ }^{13} \mathrm{CS}(2-1)$ emission is significantly weaker than $\mathrm{N}_{2} \mathrm{H}^{+}(1-0)$. Figure 8 presents an overlay of the integrated ${ }^{13} \mathrm{CS}(2-1)$ with the $\mathrm{N}_{2} \mathrm{H}^{+}(1-0)$ emission. It is interesting to note that while the main $\mathrm{mm}$ and $\mathrm{N}_{2} \mathrm{H}^{+}$peak is also detected in the ${ }^{13} \mathrm{CS}$ data, of the remaining $\mathrm{N}_{2} \mathrm{H}^{+}$cores, only peak 6 is associated with detectable ${ }^{13} \mathrm{CS}$ emission above the $3 \sigma$ level. This already indicates peculiar low abundances compared to more evolved regions, but likely also varying abundances between the different cores.

Toward the main continuum and line peak, the integrated ${ }^{13} \mathrm{CS}(2-1)$ emission of $0.042 \mathrm{Jy} \mathrm{km} \mathrm{s}^{-1}$ corresponds in the Rayleigh-Jeans limit to $0.52 \mathrm{~K} \mathrm{~km} \mathrm{~s}^{-1}$. How does that compare to total single-dish fluxes? Beuther et al. (2002) observed the $\mathrm{CS}(2-1)$ line toward that region with the IRAM $30 \mathrm{~m}$ telescope, and the measured integrated intensity of $3.3 \mathrm{~K} \mathrm{~km} \mathrm{~s}^{-1}$ corresponds to a flux of $17.6 \mathrm{Jy} \mathrm{km} \mathrm{s}^{-1}$. Assuming a typical line intensity ratio between ${ }^{12} \mathrm{CS}$ and ${ }^{13} \mathrm{CS}$ of $\sim 7$ we get an integrated ${ }^{13} \mathrm{CS}$ intensity of $2.45 \mathrm{Jy} \mathrm{km} \mathrm{s}^{-1}$ (toward the hot core NGC6334I and the much younger sibling NGC 6334I(N), the measured ratios are 8 and 6.5, Walsh et al. 2009). Therefore, the missing flux 
Table 1. Parameters of $\mathrm{N}_{2} \mathrm{H}^{+}(1-0)$ emission peaks.

\begin{tabular}{lrrrrrrrr}
\hline \hline$\#$ & $\begin{array}{r}\text { Offset }^{a} \\
"\end{array}$ & $\begin{array}{r}T_{\text {peak }} \\
\mathrm{K}\end{array}$ & $\begin{array}{r}v \\
\mathrm{~km} \mathrm{~s}^{-1}\end{array}$ & $\begin{array}{r}\Delta v \\
\mathrm{~km} \mathrm{~s}^{-1}\end{array}$ & $\begin{array}{r}N_{\mathrm{N}_{2} \mathrm{H}^{+}} \\
\times 10^{13} \mathrm{~cm}^{-2}\end{array}$ & $\begin{array}{r}M_{\text {vir }}^{b} \\
M_{\odot}\end{array}$ & $\begin{array}{r}\mathrm{av.} \rho \\
10^{5} \mathrm{~cm}^{-3}\end{array}$ \\
\hline 1 & $6.2 / 8.6$ & 2.1 & 7.7 & 1.8 & 11.9 & $6.6 \pm 0.3$ & $7.5-11.4$ & 19 \\
2 & $12.1 / 17.8$ & 0.7 & 6.9 & 0.8 & 12.7 & $1.9 \pm 0.3$ & $0.6-0.9$ & 12 \\
$3_{1}$ & $0.7 / 4.3$ & 1.5 & 7.3 & 0.5 & 7.0 & $0.9 \pm 0.1$ & $0.3-0.4$ & 5.9 \\
$3_{2}$ & $0.7 / 4.3$ & 0.4 & 8.3 & 0.8 & 16.3 & $2.1 \pm 0.5$ & $0.6-0.9$ & 14 \\
4 & $-10.1 /-9.0$ & 2.3 & 8.1 & 1.1 & 6.5 & $2.3 \pm 0.2$ & $1.3-1.9$ & 15 \\
$4 \mathrm{a}$ & $-9.9 /-5.8$ & 2.3 & 7.7 & 0.7 & 7.8 & $1.7 \pm 0.1$ & $0.5-0.7$ & 11 \\
5 & $-8.6 /-4.5$ & 2.2 & 7.7 & 0.8 & 7.1 & $1.9 \pm 0.1$ & $0.7-1.1$ & 12 \\
6 & $-5.7 / 0.7$ & 1.3 & 8.2 & 0.7 & 12.6 & $2.0 \pm 0.1$ & $0.5-0.7$ & 13 \\
7 & $-3.6 / 5.3$ & 1.3 & 8.1 & 0.6 & 5.1 & $0.7 \pm 0.1$ & $0.3-0.5$ & 4.6 \\
8 & $-2.9 / 8.2$ & 4.3 & 8.0 & 0.7 & 0.1 & $0.8 \pm 0.1$ & $0.5-0.7$ & 5.2 \\
9 & $-1.0 / 13.0$ & 0.9 & 8.2 & 0.5 & 8.3 & $0.9 \pm 0.2$ & $0.3-0.4$ & 5.9 \\
10 & $-0.1 / 17.8$ & 2.2 & 8.1 & 0.5 & 2.0 & $0.4 \pm 0.1$ & $0.3-0.5$ & 2.6 \\
$11_{1}$ & $4.6 / 23.7$ & 1.4 & 7.6 & 0.5 & 1.1 & $0.3 \pm 0.1$ & $0.3-0.5$ & 2.0 \\
$11_{2}$ & $4.6 / 23.7$ & 0.6 & 8.6 & 0.8 & 4.9 & $0.7 \pm 0.1$ & $0.6-0.9$ & 4.6 \\
12 & $-14.2 /-11.1$ & 2.0 & 7.7 & 1.2 & 5.7 & $2.0 \pm 0.2$ & $1.5-2.2$ & 13 \\
13 & $-5.4 /-7.4$ & 1.5 & 7.8 & 0.7 & 10.4 & $1.8 \pm 0.1$ & $0.5-0.7$ & 12 \\
$14_{1}$ & $-0.6 /-5.2$ & 0.5 & 7.8 & 1.0 & 10.7 & $1.9 \pm 0.2$ & $1.0-1.5$ & 12 \\
$14_{2}$ & $-0.6 /-5.2$ & 2.6 & 8.8 & 0.7 & 0.1 & $0.3 \pm 0.1$ & $0.5-0.7$ & 2.0 \\
15 & $-17.6 /-4.9$ & 2.6 & 8.9 & 0.5 & 0.9 & $0.3 \pm 0.3$ & $0.3-0.4$ & 2.0 \\
16 & $-24.5 / 3.3$ & 2.8 & 6.8 & 1.0 & 3.7 & $1.4 \pm 0.1$ & $0.9-1.4$ & 9.2 \\
17 & $-20.7 / 5.7$ & 3.1 & 6.8 & 1.0 & 0.1 & $0.6 \pm 0.2$ & $1.0-1.6$ & 3.9 \\
\hline
\end{tabular}

The subscripts associated with sources 3,11 and 14 correspond to two different velocity components required for the spectral fits.

${ }^{a}$ The offsets are given with respect to the phase center RA[J2000] $19^{\mathrm{h}} 19^{\mathrm{m}} 50.169^{\mathrm{s}}$ and Dec[J2000] $14^{\circ} 01^{\prime} 13.75^{\prime \prime}$.

${ }^{b}$ The virial masses were calculated following MacLaren et al. (1988) for $1 / r$ and $1 / r^{2}$ density distributions, resulting in the ranges of values. All source radii were approximated with half of the average $\mathrm{N}_{2} \mathrm{H}^{+}$synthesized beam of $\sim 3.1^{\prime \prime}$. Only for peak one we used a source radius of $3.5^{\prime \prime}$.

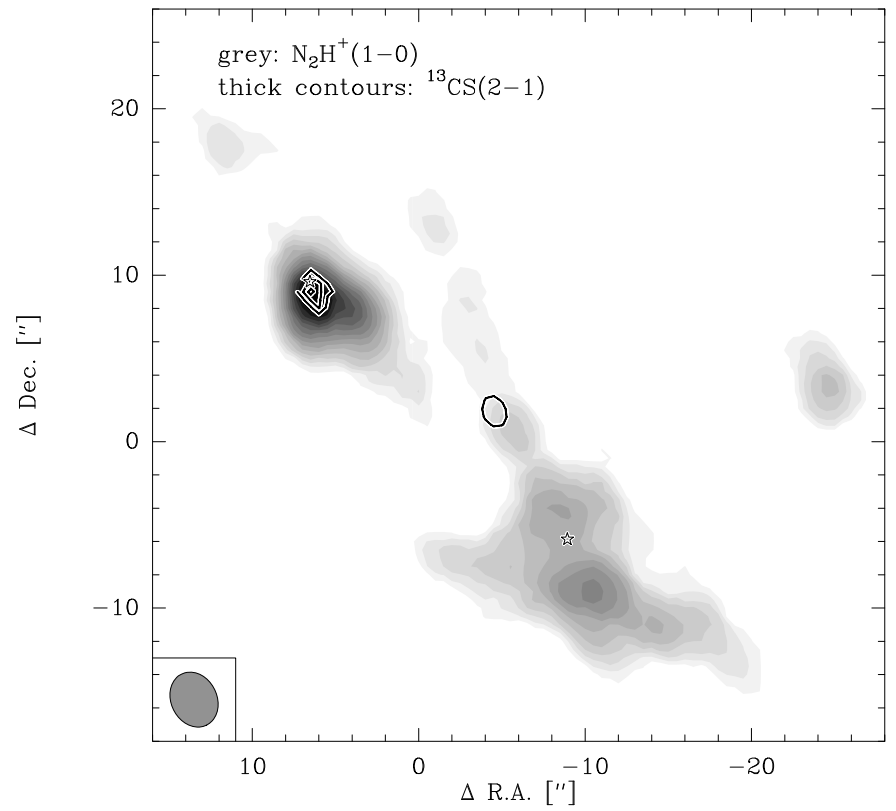

Fig. 8. The grey-scale shows the 0th moment or integrated intensity $\mathrm{N}_{2} \mathrm{H}^{+}(1-0)$ map of the isolated $\mathrm{N}_{2} \mathrm{H}^{+}(1-0)(\mathrm{F} 1, F=0,1 \rightarrow 1,2)$ hyperfine emission component as in Fig. 6. The thick contours present the ${ }^{13} \mathrm{CS}(2-1)$ emission integrated from 6.9 to $9.5 \mathrm{~km} \mathrm{~s}^{-1}$ (0th moment). The contouring is done in $3 \sigma$ steps of $5 \mathrm{mJy}_{\text {beam }}^{-1} \mathrm{~km} \mathrm{~s}^{-1}$. The stars mark the positions of the main mm continuum peaks (Figs. 1 and 3), and the synthesized beam is shown at the bottom-left. The offsets are given with respect to the phase center.

we loose by the interferometer observations amounts to approximately $80 \%$, similar to the $\sim 90 \%$ for the mm continuum data (see Sect. 3.1).

Assuming optically thin emission, we can use the ${ }^{13} \mathrm{CS}(2-1)$ data to estimate ${ }^{13} \mathrm{CS}$ column densities of the compact gas component following Rohlfs \& Wilson (2006) at the given low temperatures of $\sim 15 \mathrm{~K}$. Employing further a ${ }^{12} \mathrm{CS} /{ }^{13} \mathrm{CS}$ ratio of 50 , we can estimate the main isotopologue's ${ }^{12} \mathrm{CS}$ column densities and derive the CS abundance by comparison with the $\mathrm{H}_{2}$ column densities calculated in Sect. 3.1. This results in a CS column density of $\sim 1 \times 10^{14} \mathrm{~cm}^{-2}$ and a CS abundance with respect to $\mathrm{H}_{2}$ of $\sim 5 \times 10^{-11}$. Such very low values are consistent with chemical models that predict CS column densities of that order at the beginning of core evolution (e.g., Nomura \& Millar 2004). The estimated abundance is about 2 orders of magnitude lower than observed for example toward Orion-KL (e.g., van Dishoeck $\&$ Blake 1998), indicating CS depleting factors of $\sim 100$ in very young cores. For comparison, Zhang et al. (2009) recently found similarly high CO depletion factors (between 100 and 1000) toward the IRDC G28.32.

\subsection{Virial masses}

Following MacLaren et al. (1988), we calculated the virial masses $M_{\mathrm{vir}}=k_{2} \times R \times \Delta v^{2}$ with the constant $k_{2}$ between 190 and 126 for $1 / r$ or $1 / r^{2}$ density distributions, the core radius $R$ (in units of parsecs) and the $\mathrm{N}_{2} \mathrm{H}^{+}(1-0)$ line width $\Delta v$. For the radius we assumed almost all sources to be unresolved with an average $\mathrm{N}_{2} \mathrm{H}^{+}(1-0)$ synthesized beam of $\sim 3.1^{\prime \prime}$, corresponding to the core diameter. Only peak 1 is resolved and we used a radius of $3.5^{\prime \prime}$. Table 1 lists the range of virial masses for the given line widths and assumed density distributions.

The virial mass derived for the main core (between 7.5 and $11.4 M_{\odot}$ ) corresponds well to the gas mass derived above from the mm continuum emission (see Sect. 3.1). This indicates that the core may be in virial equilibrium. Nevertheless, since this core also exhibits the broadest line width, it is possible that the core may be in a contracting phase prior to active star formation (e.g., Caselli et al. 2002b). We doubt that the line broadening is due to an early outflow since we did not detect any $\mathrm{SiO}$ emission 
in previous observations (Beuther \& Sridharan 2007). For most other $\mathrm{N}_{2} \mathrm{H}^{+}$cores, we cannot draw conclusions regarding their virial status since most of the virial masses are at or below our $3 \sigma$ mass sensitivity of $\sim 0.9 M_{\odot}$ derived from the continuum data. Only the virial masses of the $\mathrm{N}_{2} \mathrm{H}^{+}$cores 4,10 and 12 are above this $3 \sigma$ sensitivity limit, indicating that at least these cores are stable against gravitational collapse or may even be transient, again expanding structures.

\section{Comparison with other star-forming regions}

How do the results of these two IRDCs compare with more typical regions of low-mass starless cores, for example the Pipe nebula (Alves et al. 2007; Lada et al. 2008; Rathborne et al. 2008b)? While some parameters like the masses and the majority of cores being starless are similar between the Pipe and the IRDCs discussed here, other parameters are not. For example, the mean density of the Pipe cores is $7 \times 10^{3} \mathrm{~cm}^{-3}$, approximately 2 orders of magnitude lower than what we estimate for the IRDC 19175 cores. This density difference results also in different Jeanslength of the order $0.2 \mathrm{pc}$ for the Pipe and $\sim 6000 \mathrm{AU}$ for the cores discussed here. Interestingly, like in our case where the observed core separation corresponds well to the Jeans-length, the same is the case for the Pipe nebula with a much wider average core separation of $\sim 0.26 \mathrm{pc}$ (Joao Alves, private communication). This supports the long-standing argument that the relatively simple Jeans-analysis can be considered a good approximation for the expected fragmentation scales. But why are the average densities so different? For a more conclusive answer to this question, a larger statistical sample would be required, however, the environments of the two regions already indicate a possible reason. While the Pipe nebula is relatively isolated, the IRDCs discussed in this paper are close to the intermediate- to high-mass star-forming region IRAS $19175+1357$. Therefore, it is likely that the environment - either direct influence from the more evolved IRAS source and/or the density and column density structure of the original gas clump - influences the density structure of the nearby cores and this way also determines the Jeans- and fragmentation length of the neighboring next generation of star formation.

The observed line width in typical low-mass starless cores is usually found to be close to thermal where the non-thermal line width contribution rarely exceeds the thermal part (e.g., André et al. 2007; Rathborne et al. 2008b; Foster et al. 2009). The situation is slightly different in starless cores associated with high-mass star-forming regions where on average broader line widths are found. For example, Fontani et al. (2008) found $\mathrm{N}_{2} \mathrm{H}^{+}$ line width around $0.7 \mathrm{~km} \mathrm{~s}^{-1}$ in the intermediate- to high-mass star-forming region IRAS $05345+3157$, whereas Wang et al. (2008) and Zhang et al. (2009) found $\mathrm{NH}_{3}$ line widths around $1.7 \mathrm{~km} \mathrm{~s}^{-1}$ towards the youngest massive core associated with $\mathrm{G} 28.34+0.06$.

The average line width found in the 17 cores of IRDC $19175-$ 4 and IRDC-19175-5 is $\sim 0.8 \mathrm{~km} \mathrm{~s}^{-1}$ with a spread between 0.5 and $1.8 \mathrm{~km} \mathrm{~s}^{-1}$. As discussed in Sect. 3.2, the lower end of that regime is less accurately determined since our velocity resolution is only $0.2 \mathrm{~km} \mathrm{~s}^{-1}$, barely resolving the narrowest lines. Similar to Fontani et al. (2008), our measured line widths exceed those of the low-mass cores, however, they are still narrower than observed toward more evolved massive star-forming regions (e.g., high-mass protostellar objects, HMPOs, or ultracompact HII regions, UCHIIs, Beuther et al. 2002; Churchwell et al. 1990; Cesaroni et al. 1991). This indicates a lower level of turbulent motions at the onset of intermediate-mass star formation compared to its successive evolution. Furthermore, the many $\mathrm{N}_{2} \mathrm{H}^{+}$sub-sources show that such intermediate-mass cores can fragment significantly and potentially form small groups or clusters.

Maybe a bit surprising, the line widths found toward the very young clump in G28.34+0.06 by Wang et al. (2008) are intermediate between the narrower lines we and Fontani et al. (2008) observed, whereas they are still less broad than than those of typical HMPOs or UCHII regions. It is interesting to compare a few other quantities of this particular region P1 in G28.34+0.06 (see Zhang et al. 2009): The average separation between their 5 sub-cores is $0.19 \mathrm{pc}$, similar to the Pipe and different to the case discussed here. In contrast to that, the average densities in $\mathrm{G} 28.34+0.06$ range between $10^{6}$ and $10^{7} \mathrm{~cm}^{-3}$, comparable to the values found in IRDC 19175-4 and IRDC-19175-5. As outlined above, for the Pipe nebula, densities, Jeans-length and observed core separation match rather well. This is not the case for the P1 region in G28.34+0.06. Their densities imply smaller Jeans length and lower Jeans-masses than observed. As discussed by Zhang et al. (2009), in the case of G28.34+0.06, not only thermal pressure and gravity seem to control the fragmentation of the initial gas clump, but other processes are required. The broader observed line width there indicates a significant turbulent contribution which may help to stabilize the cloud cores against further fragmentation at that evolutionary stage.

The situation for IRDC 19175-4 and IRDC-19175-5 is different to G28.34+0.06. Since here densities, Jeans-lengths and observed core separation match well, turbulence seems to play a less important role for the fragmentation processes. The cloud sub-structure can well be dominated by the interplay of mainly thermal pressure and gravity.

\section{Discussion and conclusions}

Combining Spitzer IRAC and MIPS data from 3.6 to $70 \mu \mathrm{m}$ with $\mathrm{mm}$ continuum and $\mathrm{N}_{2} \mathrm{H}^{+}$observations from the Plateau de Bure Interferometer and the IRAM $30 \mathrm{~m}$ telescope, we identify two very young gas and dust cores of intermediate mass ( $\sim 87 M_{\odot}$ together for IRDC $19175-4$ and IRDC 19175-5) in the vicinity of the intermediate- to high-mass starforming region IRAS 19175+1357. The high-spatial-resolution PdBI $3.23 \mathrm{~mm}$ continuum data clearly resolve IRDC 19175-4. However, IRDC $19175-5$ is only barely detected above the $3 \sigma$ level. More importantly, the $\mathrm{N}_{2} \mathrm{H}^{+}(1-0)$ line reveals a completely different structure with 17 separate emission sources within the vicinity of IRDC 19175-4 and IRDC 19175-5. Fitting the full $\mathrm{N}_{2} \mathrm{H}^{+}(1-0)$ hyperfine structure, we are able to derive the line parameters (intensity, peak velocity, line width and optical depth) for all cores, and from that the $\mathrm{N}_{2} \mathrm{H}^{+}$column densities and virial masses.

Of particular interest are the measured line width of the $\mathrm{N}_{2} \mathrm{H}^{+}$cores. Only the most massive core IRDC $19175-4$ with a mass of $\sim 10 M_{\odot}$ exhibits a line width of $\sim 1.8 \mathrm{~km} \mathrm{~s}^{-1}$ whereas all other $\mathrm{N}_{2} \mathrm{H}^{+}$cores have significantly narrower line widths below or around $1 \mathrm{~km} \mathrm{~s}^{-1}$. While the measured line widths between 0.5 and $1.8 \mathrm{~km} \mathrm{~s}^{-1}$ are still larger than the thermal value of $\sim 0.15 \mathrm{~km} \mathrm{~s}^{-1}$ (at $15 \mathrm{~K}$ ), the line widths are narrow compared to more evolved high-mass star-forming regions (e.g., Churchwell et al. 1990; Hatchell et al. 1998; Beuther et al. 2002) and hence indicate a low level of internal turbulence. Matching densities, Jeans-lengths and observed core separation indicate that the fragmentation process may be dominated by the interplay of mainly thermal pressure and gravity, but not so much by turbulence. These low-turbulence cores are in the vicinity of a 
high-mass star-forming region with approximately $9 \times 10^{4} \mathrm{AU}$ separation from IRAS $19175+1357$. As outlined in Sect. 1, although we cannot proof direct interaction between the more evolved IRAS source and the younger IRDCs, both should stem from the same gas clump that originally fragmented and produced (massive) star-forming regions of different evolutionary stages.

A comparison of the $\mathrm{N}_{2} \mathrm{H}^{+}$column densities with the corresponding $\mathrm{H}_{2}$ values (and upper limits) derived from the dust continuum emission, indicates significant abundance differences between the $\mathrm{N}_{2} \mathrm{H}^{+}$cores. While most of the lower-mass cores are consistent with typical $\mathrm{N}_{2} \mathrm{H}^{+}$abundances of the order $10^{-10}$ with respect to $\mathrm{H}_{2}$, the main core IRDC $19175-4$ has lower abundances of the order $10^{-11}$. Since this source also shows the broadest line width, we interprete this lowered abundances as likely due to higher densities from the contracting core which increases the recombination rate and hence lowers the abundances of the ions. Furthermore, the weak ${ }^{13} \mathrm{CS}(2-1)$ detection allows us to estimate CS column densities and abundances, and we find CS depletion factors of the order 100 compared to more evolved regions like Orion-KL.

The virial analysis and line width measurements indicate that the main core IRDC 19175-4, although being close to virial equilibrium, may already be in a phase of core contraction and hence at the verge of star formation. For the remaining cores, the status can be different. Since we have only upper limits for the gas masses from the dust continuum emission, a real virial analysis is difficult, however, these upper limits are consistent with virial stable cores. Similarly, the separation between the different subcores corresponds approximately to the Jeans-length at $15 \mathrm{~K}$ and a density of $10^{6} \mathrm{~cm}^{-3}$. Hence, the cores can be stable against further fragmentation. All this indicates that many of them may never enter star formation activity but could rather be transient or stable cores in the vicinity of other massive star-forming regions.

In summary, here we report the characterization of 17 lowto intermediate mass $\mathrm{N}_{2} \mathrm{H}^{+}$cores with very low levels of turbulence in the vicinity of a high-mass star-forming region. The non-detection up to $70 \mu \mathrm{m}$ wavelength, the low levels of turbulence as well as no other signs of star formation in previous studies indicates that we are dealing with starless cores. While some of them maybe contracting and may start active star formation in the future, many of them may be stable against collapse and hence potentially even only transient structures. The average densities are approximately 2 orders of magnitude higher than in the Pipe nebula which is also manifested in smaller core separations. We note that these rather quiescent cores are spatially associated with more turbulent intermediate- to high-mass starforming cores, implying that low and high turbulence regions can co-exist in relatively close proximity.

Acknowledgements. Thanks a lot to Helmut Dannerbauer and Roy van Boekel for helping with the Spitzer data. Furthermore, we like to thank C. de Vries for providing the $\mathrm{N}_{2} \mathrm{H}^{+}$column density calculation routine. In addition, we appreciated a lot the referee's comments improving the paper. H.B. acknowledges financial support by the Emmy-Noether-Program of the Deutsche Forschungsgemeinschaft (DFG, grant BE2578).

\section{References}

Aikawa, Y., Ohashi, N., \& Herbst, E. 2003, ApJ, 593, 906

Allen, L. E., Calvet, N., D’Alessio, P., et al. 2004, ApJS, 154, 363

Alves, J., Lombardi, M., \& Lada, C. J. 2007, A\&A, 462, L17

André, P., Belloche, A., Motte, F., \& Peretto, N. 2007, A\&A, 472, 519

Bacmann, A., André, P., Puget, J.-L., et al. 2000, A\&A, 361, 555

Benjamin, R. A., Churchwell, E., Babler, B. L., et al. 2003, PASP, 115, 953

Bergin, E. A., \& Langer, W. D. 1997, ApJ, 486, 316

Bergin, E. A., Alves, J., Huard, T., \& Lada, C. J. 2002, ApJ, 570, L101
Beuther, H., \& Sridharan, T. K. 2007, ApJ, 668, 348

Beuther, H., \& Steinacker, J. 2007, ApJ, 656, L85

Beuther, H., Schilke, P., Menten, K. M., et al. 2002, ApJ, 566, 945

Beuther, H., Sridharan, T. K., \& Saito, M. 2005, ApJ, 634, L185

Beuther, H., Churchwell, E. B., McKee, C. F., \& Tan, J. C. 2007, in Protostars and Planets V, ed. B. Reipurth, D. Jewitt, \& K. Keil, 165

Birkmann, S. M., Krause, O., \& Lemke, D. 2006, ApJ, 637, 380

Bonnell, I. A., Larson, R. B., \& Zinnecker, H. 2007, in Protostars and Planets V, ed. B. Reipurth, D. Jewitt, \& K. Keil, 149

Carey, S. J., Noriega-Crespo, A., Price, S. D., et al. 2005, AAS Meeting Abstracts, 207, 63.33

Carey, S. J., Noriega-Crespo, A., Mizuno, D. R., et al. 2009, PASP, 121, 76

Caselli, P., Myers, P. C., \& Thaddeus, P. 1995, ApJ, 455, L77

Caselli, P., Benson, P. J., Myers, P. C., \& Tafalla, M. 2002a, ApJ, 572, 238

Caselli, P., Walmsley, C. M., Zucconi, A., et al. 2002b, ApJ, 565, 331

Cesaroni, R., Walmsley, C. M., Koempe, C., \& Churchwell, E. 1991, A\&A, 252, 278

Cesaroni, R., Galli, D., Lodato, G., Walmsley, C. M., \& Zhang, Q. 2007, in Protostars and Planets V, ed. B. Reipurth, D. Jewitt, \& K. Keil, 197

Chen, X., Launhardt, R., \& Henning, T. 2007, ApJ, 669, 1058

Churchwell, E., Walmsley, C. M., \& Cesaroni, R. 1990, A\&AS, 83, 119

Churchwell, E., Babler, B. L., Meade, M. R., et al. 2009, PASP, 121, 213

Draine, B. T., Dale, D. A., Bendo, G., et al. 2007, ApJ, 663, 866

Egan, M. P., Shipman, R. F., Price, S. D., et al. 1998, ApJ, 494, L199

Fang, M., van Boekel, R., Wang, M., et al. 2009, A\&A accepted, [arXiv:0907.2380]

Fazio, G. G., Hora, J. L., Allen, L. E., et al. 2004, ApJS, 154, 10

Fontani, F., Caselli, P., Bourke, T. L., Cesaroni, R., \& Brand, J. 2008, A\&A, 477, L45

Forbrich, J., Schreyer, K., Posselt, B., Klein, R., \& Henning, T. 2004, ApJ, 602, 843

Forbrich, J., Stanke, T., Klein, R., et al. 2009, A\&A, 493, 547

Foster, J. B., Rosolowsky, E. W., Kauffmann, J., et al. 2009, ApJ, 696, 298

Frerking, M. A., Langer, W. D., \& Wilson, R. W. 1982, ApJ, 262, 590

Hatchell, J., Thompson, M. A., Millar, T. J., \& MacDonald, G. H. 1998, A\&AS, 133,29

Hildebrand, R. H. 1983, QJRAS, 24, 267

Hoare, M. G., Kurtz, S. E., Lizano, S., Keto, E., \& Hofner, P. 2007, in Protostars and Planets V, ed. B. Reipurth, D. Jewitt, \& K. Keil, 181

Jackson, J. M., Finn, S. C., Rathborne, J. M., Chambers, E. T., \& Simon, R. 2008, ApJ, 680, 349

Jenkins, E. B. 2004, in Origin and Evolution of the Elements, ed. A. McWilliam, \& M. Rauch, 336

Klein, R., Posselt, B., Schreyer, K., Forbrich, J., \& Henning, T. 2005, ApJS, 161, 361

Lada, C. J., Muench, A. A., Rathborne, J., Alves, J. F., \& Lombardi, M. 2008, ApJ, 672, 410

MacLaren, I., Richardson, K. M., \& Wolfendale, A. W. 1988, ApJ, 333, 821

Mathis, J. S., Rumpl, W., \& Nordsieck, K. H. 1977, ApJ, 217, 425

Nomura, H., \& Millar, T. J. 2004, A\&A, 414, 409

Ormel, C. W., Shipman, R. F., Ossenkopf, V., \& Helmich, F. P. 2005, A\&A, 439, 613

Ossenkopf, V., \& Henning, T. 1994, A\&A, 291, 943

Pillai, T., Wyrowski, F., Menten, K. M., \& Krügel, E. 2006, A\&A, 447, 929

Rathborne, J. M., Jackson, J. M., Chambers, E. T., et al. 2005, ApJ, 630, L181

Rathborne, J. M., Jackson, J. M., \& Simon, R. 2006, ApJ, 641, 389

Rathborne, J. M., Jackson, J. M., Zhang, Q., \& Simon, R. 2008a, ApJ, 689, 1141

Rathborne, J. M., Lada, C. J., Muench, A. A., Alves, J. F., \& Lombardi, M. 2008b, ApJS, 174, 396

Rieke, G. H., Young, E. T., Engelbracht, C. W., et al. 2004, ApJS, 154, 25

Rohlfs, K., \& Wilson, T. L. 2006, Tools of radio astronomy, 4th rev. and enl. ed., K. Rohlfs and T.L. Wilson (Berlin: Springer)

Simon, R., Jackson, J. M., Rathborne, J. M., \& Chambers, E. T. 2006, ApJ, 639, 227

Sridharan, T. K., Beuther, H., Schilke, P., Menten, K. M., \& Wyrowski, F. 2002, ApJ, 566, 931

Sridharan, T. K., Beuther, H., Saito, M., Wyrowski, F., \& Schilke, P. 2005, ApJ, 634, L57

Stahler, S. W., \& Palla, F. 2005, The Formation of Stars, ed. S. W. Stahler, F. Palla (Wiley-VCH, 2005), 865

Tafalla, M., \& Santiago, J. 2004, A\&A, 414, L53

Tafalla, M., Myers, P. C., Caselli, P., \& Walmsley, C. M. 2004, A\&A, 416, 191 van Dishoeck, E. F., \& Blake, G. A. 1998, ARA\&A, 36, 317

Walsh, A.J., Thorwirth, S., Beuther, H., Burton, M.G. 2009, MNRAS, submitted Wang, Y., Zhang, Q., Pillai, T., Wyrowski, F., \& Wu, Y. 2008, ApJ, 672, L33

Werner, M. W., Roellig, T. L., Low, F. J., et al. 2004, ApJS, 154, 1

Zhang, Q., Wang, Y., Pillai, T., \& Rathborne, J. 2009, ApJ, 696, 268

Zinnecker, H., \& Yorke, H. W. 2007, ARA\&A, 45, 481 